\title{
๖The Poisson Link between Internal Wave and Dissipation Scales in the Thermocline. Part II: Internal Waves, Overturns, and the Energy Cascade
}

\author{
ROBERT PINKEL ${ }^{\mathrm{a}}$ \\ ${ }^{a}$ Marine Physical Laboratory, Scripps Institution of Oceanography, University of California, San Diego, La Jolla, California
}

(Manuscript received 25 November 2019, in final form 30 August 2020)

\begin{abstract}
The irregular nature of vertical profiles of density in the thermocline appears well described by a Poisson process over vertical scales $2-200 \mathrm{~m}$. To what extent does this view of the thermocline conflict with established models of the internal wavefield? Can a one-parameter Poisson subrange be inserted between the larger-scale wavefield and the microscale field of intermittent turbulent dissipation, both of which require many parameters for their specification? It is seen that a small modification to the Poisson vertical correlation function converts it to the corresponding correlation function of the Garrett-Munk (GM) internal wave spectral model. The linear scaling relations and vertical wavenumber dependencies of the GM model are maintained provided the Poisson constant $\kappa_{0}$ is equated with the ratio of twice the displacement variance to the vertical correlation scale of the wavefield. Awareness of this Poisson wavefield relation enables higher-order strain statistics to be determined directly from the strain spectrum. Using observations from across the Pacific Ocean, the average Thorpe scale of individual overturning events is found to be nearly equal to the inverse of $\kappa_{0}$, the metric of background thermocline distortion. If the fractional occurrence of overturning $\phi$ is introduced as an additional parameter, a Poisson version of the Gregg-Henyey relationship can be derived. The Poisson constant, buoyancy frequency, and $\phi$ combine to create a complete parameterization of energy transfer from internal wave scales through the Poisson subrange to dissipation. An awareness of the underlying Poisson structure of the thermocline will hopefully facilitate further improvement in both internal wave spectral models and ocean mixing parameterizations.
\end{abstract}

KEYWORDS: Internal waves; Turbulence; Wave breaking; Diapycnal mixing; Thermocline; Profilers, oceanic

\section{Introduction}

Walter Munk concluded his classic 1981 review (Munk 1981) of internal waves with a speculation on the cascade of energy through the wave spectrum and its relation to wave breaking and the diapycnal buoyancy flux in the thermocline. He divided the wavefield into intrinsic waves that propagate quasi-linearly, undisturbed by their neighbors, and compliant waves whose speed of propagation is so slow that they are strongly affected by the larger-scale wavefield. Munk felt that the boundary between these two types of waves, at vertical scale $k_{c}^{-1}$, should somehow be related to the cutoff in vertical wavenumber spectra found at roughly $10-\mathrm{m}$ scale. It was puzzling that this "10-m cutoff" appeared to be independent of depth and buoyancy frequency, in violation of WKB scaling. Existing data also indicated that the internal wave spectrum had a quasiuniversal level. He identified a parameter of nonlinearity and felt that the point at which the wavefield became highly nonlinear should determine both $k_{c}$ and the universal spectral level. However, his nonlinearity criterion only specified the product of cutoff wavenumber and level of the shear or strain spectrum (which is white in vertical wavenumber), rather than identifying both of these key parameters independently. He concluded with the optimistic hope that "we were close to having the various pieces fall into place."

¿ Denotes content that is immediately available upon publication as open access.

Corresponding author: Robert Pinkel, rpinkel@ucsd.edu
Five years later, Henyey et al. (1986) published a modeling study showing that energy cascaded through the internal wave spectrum at a rate proportional to the spectral level squared. ${ }^{1}$ If the spectral level is not universal, small changes in level should result in large changes in dissipation. Shortly thereafter, Gregg (1989) observationally verified Henyey's result and produced a predictive model for dissipation based on the measurement of the finescale shear. His model was subsequently confirmed and refined by Polzin et al. (1995), Hibiya et al. (2012), Polzin et al. (2014), and others and has become the accepted standard for parameterizing deep ocean mixing. Müller et al. (1992), demonstrated that the product of cutoff wavenumber and spectral level indeed appeared to be constant in vertical wavenumber spectra of shear, supporting Munk's earlier groundwork.

In Pinkel (2020, hereafter Part I), it was demonstrated that a Poisson statistical model has great skill in replicating both probability density functions and power spectra of vertical strain in the thermocline. Indeed, the relationship between cutoff wavenumber $\kappa_{0}\left(\mathrm{rad} \mathrm{m}^{-1}\right)$ and spectral level $\kappa_{0}^{-1}$ satisfies Munk's nonlinear criterion in the apparent absence of dynamical considerations. The present challenge is to link the

\footnotetext{
${ }^{1}$ Müller and Olbers (1975) were the first to derive a nonlinear transfer rate through the spectrum that was proportional to the square of the spectral energy level [their Eq. (37) and preceding]. The cascade was one of a number of interaction processes they considered. McComas and Muller (1981a,b) both considered scenarios where the downscale transfer of energy was proportional to spectral level squared. I am indebted to a reviewer for pointing this out.
} 
Poisson model of the finescale thermocline (Pinkel and Anderson 1992) to the larger-scale Gaussian internal wavefield and to the small-scale processes that dissipate energy in the sea.

The problem is distantly analogous to that of replacing the equations that describe the molecular dynamics of an ideal gas with a simple diffusion equation that treats the behavior of an aggregate of molecules statistically. Here, the focus is on depth variability alone, not space and time together, and the task is somewhat simpler. Can the single Poisson parameter $\kappa_{0}$ be related to the numerous internal wave and turbulence-scale variables in a physically plausible manner?

Initially, data-derived second- and third-order structure functions are presented and compared with both Poisson (Pinkel and Anderson 1992; Part I) and internal wave models. The Poisson model structure functions begin to diverge from observations at large vertical separations, where internal wave vertical displacement, as well as strain, becomes uncorrelated. The internal wave displacement correlation scale $Z_{\text {corr_sL }}$, of order $100-300 \mathrm{~m}$, defines the outer boundary of the Poisson subrange. A small modification to the Poisson vertical displacement covariance function corrects the divergent behavior, simultaneously replicating the covariance of the Garrett-Munk (GM) model of the internal wave spectrum, Munk (1981). Merging the GM and Poisson models enables the determination of non-Gaussian quantities such as finescale skewness and kurtosis from GM internal wavescale parameters. The desired link between the many parameters of GM and the single Poisson parameter $\kappa_{0}$ is uncovered.

Shifting attention to dissipation scales, the Poisson parameter $\kappa_{0}$ is shown to be strongly correlated with the vertical extent of observed overturning events (the Thorpe scale; Thorpe 1977; Dillon 1982). The Gregg-Henyey parameterization can then be recast in terms of $\kappa_{0}$ and internal-wave-scale quantities. The essential levels and dependencies of the parameterization are recovered without need for arbitrary tuning factors. The Poisson subrange thus fits consistently in the center of the energy cascade, adding a non-Gaussian perspective to our present understandings.

\section{The structure function of the Poisson thermocline}

The data considered are vertical profiles of ocean density obtained in seven Pacific Ocean experiments, as described in Part I of this work. There, it is demonstrated that the vertical straining of the thermocline appears to be well modeled as a Poisson process over vertical scales 2-200 m. In linking this non-Gaussian domain with the larger-scale world of Munk's intrinsic wavefield, structure functions of vertical displacement prove to be a surprisingly useful metric.

The structure function is a function of separation scale $\overline{\Delta z}$ and is defined as

$$
\begin{aligned}
M_{\eta}^{n}(\overline{\Delta z}) & \equiv\left\langle\Delta \hat{\eta}^{n}[\eta(z)-\eta(z-\overline{\Delta z})-\overline{\Delta z}]^{n}\right\rangle \\
& =\left\langle[\hat{\eta}(z)-\hat{\eta}(z-\overline{\Delta z})]^{n}\right\rangle=\overline{\Delta z}^{n}\left\langle[\gamma(\overline{\Delta z})-1]^{n}\right\rangle
\end{aligned}
$$

Here, $\hat{\eta} \equiv \eta-\bar{\eta}$ is the displacement of a density surface from its mean position.

Second-order descriptors such as the power spectrum $S_{\eta}(k)$ and the autocorrelation function $\hat{R}_{\eta}(\overline{\Delta z}) \equiv\langle\hat{\eta}(z) \hat{\eta}(z-\overline{\Delta z})\rangle /\left\langle\hat{\eta}^{2}\right\rangle$ are more commonly used in oceanography than structure functions. These are related to the second-order structure function by

$$
M_{\eta}^{2}(\overline{\Delta z})=2\left\langle\hat{\eta}^{2}\right\rangle\left[1-\hat{R}_{\eta}(\overline{\Delta z})\right]=2 \int_{-\infty}^{\infty} S_{\eta}(k) \sin ^{2}(k \overline{\Delta z} / 2) d k
$$

At scales $\overline{\Delta z}>\kappa_{0}^{-1}$, the moments of the gamma PDF are

$$
\begin{aligned}
& M_{\eta}^{2}(\overline{\Delta z})=\overline{\Delta z} / \kappa_{0}, \\
& M_{\eta}^{3}(\overline{\Delta z})=2 \overline{\Delta z} / \kappa_{0}^{2},
\end{aligned}
$$

with skewness

$$
\zeta(\overline{\Delta z}) \equiv M_{\eta}^{3}(\overline{\Delta z}) /\left[M_{\eta}^{2}(\overline{\Delta z})\right]^{3 / 2}=2 /\left(\kappa_{0} \overline{\Delta z}\right)^{1 / 2} .
$$

Estimates of the second- and third-order structure functions of strain are formed from observations in overlapping 200-m-depth bins that match those for the spectra of Fig. 6 in Part I. The Hawaii Ocean-Mixing Experiment (HOME) Nearfield and Farfield data are presented in Fig. 1, with a summary of all cruises given in appendix A (Figs. A1 and A2). An assumed noise variance is subtracted from the second-order structure function (Part I, Table 1). A small positive "noise mean-cube," equivalent to 10 times the (noise variance) $)^{3 / 2}$ is subtracted from the third-order structure function estimate, as well. Oddly, the corrections that seem appropriate, given the shape of the curves, are smaller than would be predicted from a reasonable estimate of our precision in measuring the depth of a density surface. It is likely that the error in isopycnal depth estimation is correlated over small vertical separations, given the 2-m lowpass filter that is applied to the raw temperature and conductivity profiles. Similarly, since isopycnals are constrained from passing through one another, the noise contribution to the mean cube must be a positive number. ${ }^{2}$ The noise correction applied to the third-order structure function is negligible at vertical scales greater than $4 \mathrm{~m}$.

Estimates of the structure functions (Figs. 1a and 1b) are generally consistent with the Poisson model. Note that the single constant $\kappa_{0}$, associated with conceptual Poisson elements of 1-2-m vertical scale, governs the behavior of the second-order structure functions to scales of 30-100 m, even though the associated probability density functions of separation visually appear to be Gaussian at much smaller separations (Part I, Fig. 3). The lack of depth variability in the second-order structure function $M_{\eta}^{2}(\overline{\Delta z})$ for the HOME Farfield site is impressive, and is consistent with the depth independence of $\kappa_{0}$ in the open ocean. The displacement variance atop Kaena Ridge in the HOME Nearfield is similar to that in the Farfield $(450 \mathrm{~km}$ to the southwest) in the upper ocean. However, for the Nearfield, a generation site for the baroclinic tide, both $M_{\eta}^{2}(\overline{\Delta z})$ and $M_{\eta}^{3}(\overline{\Delta z})$ grow with depth at fixed separation $\overline{\Delta z}$,

\footnotetext{
${ }^{2}$ If isopycnals are constrained to not cross, negative strain errors are bounded by -1 . Positive strain errors can be arbitrarily large, in principle.
} 

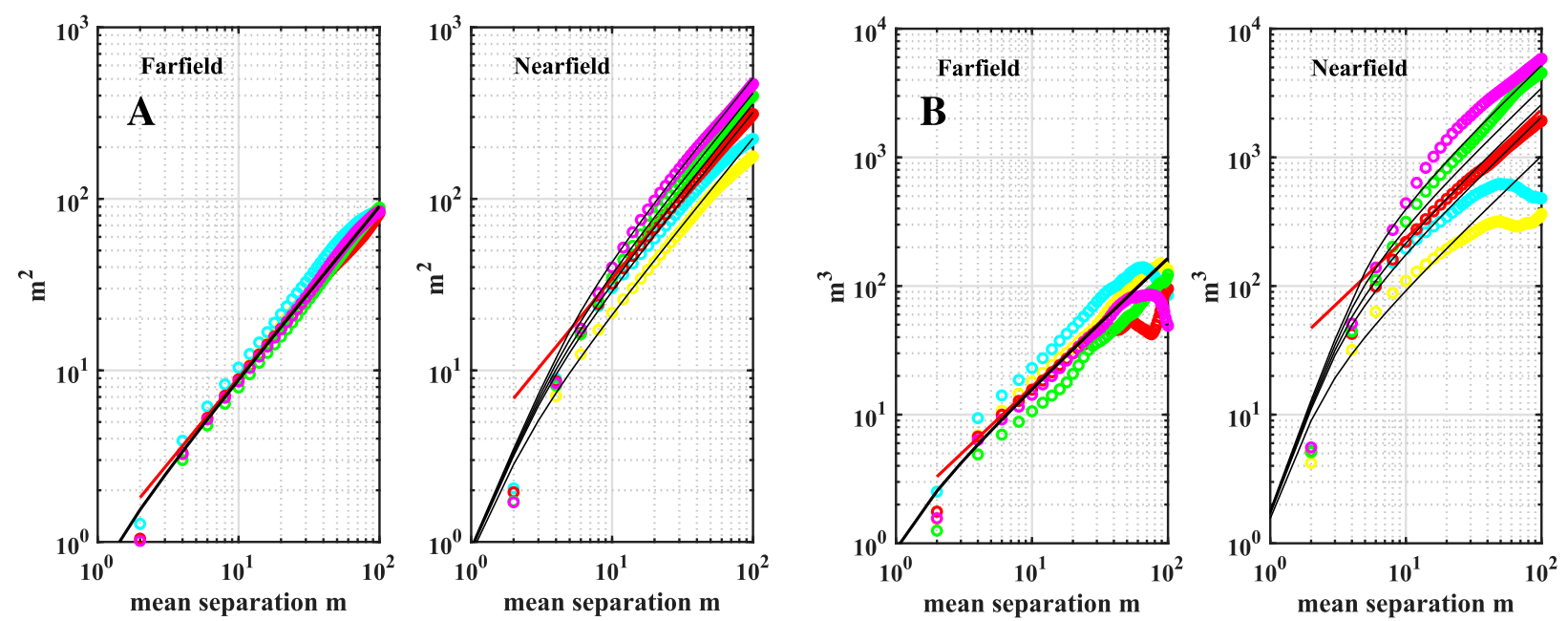

FIG. 1. A comparison of (a) second- and (b) third-order structure functions at the open-ocean Farfield site and the seafloor-intensified Nearfield site. The various colors represent different $200-\mathrm{m}$ depth intervals, overlapped by $100 \mathrm{~m}$. The red reference line gives the Poisson model prediction while the black lines give Poisson microscale model results (Part I, appendix C). A single value of $\kappa_{0}$ well represents all observations at the Farfield open-ocean site. In the Nearfield, as well as at other sites with vertically inhomogeneous wave activity, $\kappa_{0}$ varies. Structure functions for all cruises are presented in appendix A.

corresponding to a decrease in $\kappa_{0}$ as the seafloor is approached. The structure function $M_{\eta}^{2}(\overline{\Delta z})$ increases by about a factor of 3 over the depth intervals from 200-400 to 600-800 m while $M_{\eta}^{3}(\overline{\Delta z})$ increases by nearly a factor of 10 , consistent with the $\kappa_{0}^{-2}$ dependence (3) of the Poissongamma PDF.

The increase in $M_{\eta}^{3}(\overline{\Delta z})$ with increasing separation represents an interesting competition between the growing expected magnitude of the random variable $\Delta \hat{\eta}$ and the progression of its associated PDF toward a Gaussian form. The value of $\overline{\Delta z}$ at which $M_{\eta}^{3}(\overline{\Delta z})$ attains its maximum is a potential definition of the boundary between the intrinsic and compliant constituents of the wavefield, Munk's $k_{c}^{-1}$. An alternative definition of $k_{c}^{-1}$ is set by the maximum of the ratio of $M_{\eta}^{3}(\overline{\Delta z}) / M_{\eta}^{2}(\overline{\Delta z})$. Both should be examined routinely in field data.

At scales less than $10 \mathrm{~m}$, the observational estimates of $M_{\eta}^{2}(\overline{\Delta z})$ clearly fall below the $(\overline{\Delta z}) / \kappa_{0}$ reference associated with the gamma PDF. The discrepancy is most pronounced in the highly strained, low $\kappa_{0}$ sites. The departure of $M_{\eta}^{3}(\overline{\Delta z})$ from its gamma model form at small $\overline{\Delta z}$ is striking as well. This departure extends to larger scales, $10-20 \mathrm{~m}$. It is apparent in all datasets to a degree proportional to the strain variance $\left(\sim \kappa_{0}^{-1}\right)$. While finite sensor resolution plays a role, a more fundamental issue is involved, stemming from the tracking of humanimposed tracers (reference densities) on a vertical stack of unknowable Poisson elements. Theoretical predictions for the structure functions of imposed tracers, based on an underlying Poisson structure for the thermocline, are derived in appendix C of Part I (black lines, Fig. 1). They demonstrate rather remarkable agreement with the data, particularly at energetic sites such as the HOME Nearfield. At such sites, small values of $\kappa_{0}$ shift this "microscale" behavior to sufficiently large vertical scale that finite sensor resolution does not overly contaminate its signature.

\section{The Poisson wavefield relation}

The second-order structure function $M_{\eta}^{2}(\overline{\Delta z})$ provides the conceptual link between the Poisson subrange and a largerscale Gaussian thermocline populated by intrinsic internal waves. The structure functions for the open-ocean sites each collapse (e.g., HOME Farfield, Fig. 1) to a depth-independent curve (2), in spite of large variations in buoyancy frequency with depth. For linear waves in the WKB approximation, wave amplitude grows and vertical wavenumber decreases as the buoyancy frequency decreases with depth. A perhaps underappreciated fact is that these two effects evolve in concert such that the WKB second-order structure function of vertical displacement is invariant with $\operatorname{depth} / N^{2}(z)$ for both the compliant wavefield and the Poisson subrange, rendering it an exceptionally useful metric of the state of the thermocline.

This is seen by recalling (2):

$$
M_{\eta}^{2}(\overline{\Delta z})=\left\langle[\hat{\eta}(z)-\hat{\eta}(z-\overline{\Delta z})]^{2}\right\rangle=2\left\langle\hat{\eta}^{2}\right\rangle\left[1-\hat{R}_{\eta}(\overline{\Delta z})\right] .
$$

At small separations the autocorrelation can be approximated by the leading terms in its Taylor expansion:

$$
\hat{R}_{\eta}(\overline{\Delta z}) \approx 1-|\overline{\Delta z}| / Z_{\text {corr_sL }}+\cdots,
$$

where $Z_{\text {corr_sL }} \equiv\left(d \hat{R}_{\eta} /\left.d \overline{\Delta z}\right|_{0}\right)^{-1}$ defines the vertical correlation scale of vertical displacement $\hat{\eta}$. For linear internal waves in the WKB approximation,

$$
\left\langle\hat{\eta}^{2}\right\rangle=\left\langle\hat{\eta}_{0}^{2}\right\rangle N_{0} / N(z)
$$

and

$$
Z_{\text {corr_sL }}=Z_{\text {corr_o } 0} N_{0} / N(z) \text {. }
$$

Here, $Z_{\text {corr_o } 0},\left\langle\hat{\eta}_{0}^{2}\right\rangle$, and $N_{0}$ are measured at a common reference depth. 

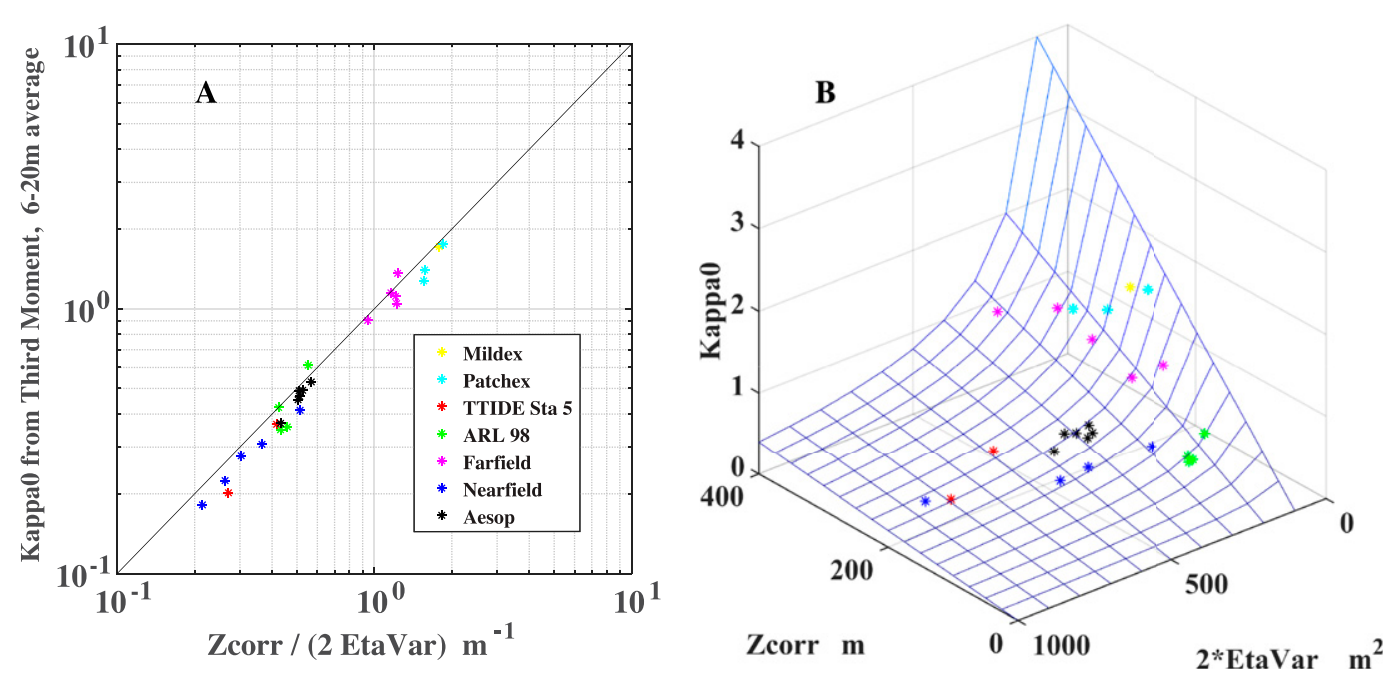

FIG. 2. The Poisson wavefield relation. (a) The ratio of the vertical correlation scale of the wavefield to twice the displacement variance, key descriptors of the second-order wavefield, is compared with estimates of $\kappa_{0}$ determined from the third moment of isopycnal separation. Colored dots represent a $200-\mathrm{m}$ vertical average, overlapped by $100 \mathrm{~m}$. (b) The variability of $Z_{\text {corr_sL }}, 2\left\langle\hat{\eta}^{2}\right\rangle$, and $\kappa_{0}$ for the seven cruises is given independently, with the blue mesh surface indicating the analytic relation.

Considering variability over the full water column, we see

$M_{\eta}^{2}(\overline{\Delta z})=\overline{\Delta z} / \kappa_{0}=2\left\langle\hat{\eta}^{2}\right\rangle \overline{\Delta z} / Z_{\text {corr_sL }}=2\left\langle\hat{\eta}_{0}^{2}\right\rangle \overline{\Delta z} / Z_{\text {corr_o }}$,

independent of measurement or reference depth.

The invariance of $M_{\eta}^{2}(\overline{\Delta z})$ with changing $N^{2}$ is thus consistent with both the Poisson model and a linear internal wavefield under WKB scaling. However, in order for the Poisson model to match the correlation-based description of the structure function, the Poisson constant $\kappa_{0}$ must be related to internal wave parameters $\left\langle\hat{\eta}^{2}\right\rangle$ and $Z_{\text {corr_sL }}$ such that

$$
\kappa_{0}^{-1}=2\left\langle\hat{\eta}^{2}\right\rangle / Z_{\text {corr_sL }} .
$$

Equation (10), henceforth referred to as the Poisson wavefield relation, is the link between our familiar second-order descriptors of the wavefield and a description that applies at all orders. The fact that the Poisson and the correlation-based models for $M_{\eta}^{2}(\overline{\Delta z})$ are congruent enables a smooth transition between the models across a common domain of validity. At scales $\Delta z<Z_{\text {corr_sL }}$ the Poisson microscale and finescale predictions for $M_{\eta}^{2}(\overline{\Delta z})$ and $M_{\eta}^{3}(\overline{\Delta z})$ clearly follow the observations (Figs. 1a,b), while $M_{\eta}^{3}(\overline{\Delta z}) \equiv 0$ in a Gaussian model. In turn, as $\Delta z$ increases beyond $Z_{\text {corr_sL }}$, the Poisson model predicts that $M_{\eta}^{2}(\overline{\Delta z})$ should increase without bound. The second-order correlation-based representation of $M_{\eta}^{2}(\overline{\Delta z})$ asymptotes to $2\left\langle\hat{\eta}^{2}\right\rangle$ as $\hat{R}_{\eta}$ vanishes (2).

In Fig. 2, the accuracy of the Poisson wavefield relation is examined across the combined pan-Pacific dataset. Individual points represent a $200-\mathrm{m}$ vertical depth average, with successive averages offset vertically by $100 \mathrm{~m}$, such that there is $100 \mathrm{~m}$ of overlap between averages. The shallowest depth interval in each experiment is $100-300 \mathrm{~m}$, except in Tasman Tidal Dissipation Experiment (TTIDE), where it is $1350-1550 \mathrm{~m}$.
The Poisson model structure function (2) implies a displacement correlation

$$
\hat{R}_{\eta P}(\overline{\Delta z})=1-\frac{|\overline{\Delta z}|}{2 \kappa_{0}\left\langle\hat{\eta}^{2}\right\rangle}=1-|\overline{\Delta z}| / Z_{\text {cor__sL }}
$$

at small separations. Values of $Z_{\text {corr_sL }}$ are determined for Fig. 2 by finding the vertical separation $\overline{\Delta z}_{0.9}$ where the correlation function has value 0.9 in each vertical average. From (11), one sees that $Z_{\text {corr_sL }}=10 \overline{\Delta z}_{0.9}$.

The impressive agreement in Fig. 2 is in part a consequence of basic geometry, with $2 \kappa_{0}$ being the decay rate of an initially triangular covariance function of height $\hat{\eta}^{2}$. However, $\kappa_{0}$ has a distinctly non-Gaussian identity, and is here estimated from the third moment of isopycnal separation. This demonstrates the link between the higher-order moments of the oceanic strain field and conventional second-order metrics.

\section{The intrinsic internal wave spectrum}

In presenting the internal wave spectrum, it is convenient to consider the vertical displacement covariance function, rather than the correlation function discussed previously. These are related by $R_{\eta}(\overline{\Delta z})=\left\langle\hat{\eta}^{2}\right\rangle \hat{R}_{\eta}(\overline{\Delta z})$. Extrapolating to large scales, the Poisson model covariance passes through zero at $\overline{\Delta z}=Z_{\text {corr_sL }}$ and becomes increasingly negative thereafter. The relevant observations of $M_{\eta}^{2}(\overline{\Delta z})$ depart from this model as scales approach $\overline{\Delta z} \approx Z_{\text {corr_sL. }}$. The Poisson covariance can be modified slightly to bring its behavior in accord with observations across all scales. Consider the covariance

$$
R_{\eta \mathrm{GM}}(\overline{\Delta z})=\left\langle\hat{\eta}^{2}\right\rangle e^{-|\overline{\Delta z}| / Z_{\text {cor_sLL }}},
$$

for example, which coincides with $R_{\eta} P$ for $\overline{\Delta z} \ll Z_{\text {corr_sL. }}$. 
Its associated vertical wavenumber spectrum is

$$
\begin{aligned}
& S_{\eta \mathrm{GM}}(k)=\kappa_{0}^{-1}\left\{\frac{1}{\left[\left(2\left\langle\hat{\eta}^{2}\right\rangle \kappa_{0}\right)^{-2}+(2 \pi k)^{2}\right]}\right\},-\infty<k<\infty, \\
& S_{\eta \mathrm{GM}}(k)=\frac{2\left\langle\hat{\eta}^{2}\right\rangle}{Z_{\text {corr_sL }}}\left\{\frac{1}{\left[Z_{\text {corr_sL }}^{-2}+(2 \pi k)^{2}\right]}\right\} \\
& S_{\eta \mathrm{GM}}(k)=\frac{b^{3} E N_{0}^{2}}{j_{*} \pi N(z)^{2}}\left\{\frac{\beta_{*}^{2}}{\left[\beta_{*}^{2}+(2 \pi k)^{2}\right]}\right\} .
\end{aligned}
$$

Identifying the Garrett and Munk (1972, 1975) and Munk (1981) parameters $\beta_{*}=(\pi / b) j_{*}\left[N(z) / N_{0}\right]$ with

$$
\beta_{*}=\left(2\langle\hat{\eta}\rangle^{2} \kappa_{0}\right)^{-1}=Z_{\text {corr_sL }} \mathrm{sL}^{-1},
$$

and

$$
\hat{\eta}^{2}=\frac{b^{2} E N_{0}}{2 N(z)},
$$

we recover the Munk (1981) form of the Garrett-Munk vertical wavenumber spectrum [see Gregg and Kunze 1991, their Eq. (A18)]. Equations (13a)-(13c) are identical, with (13a) giving the spectrum in terms of Poisson/compliant wavefield parameters, (13b) in terms of intrinsic wavefield parameters, and (13c) using the GM parameter set. The vertical wavenumber $k$ is in cycles per meter and the spectrum is presented in two-sided form, $-\infty<k<\infty$, such that its level is half that typically stated for $0<k<\infty$. The term $E=6.3 \times 10^{-5}$ is the Garrett-Munk dimensionless energy parameter, $b=1.3 \mathrm{~km}$ is the scale depth of the thermocline, and $j_{*}$ is the parameterized bandwidth of the spectrum, expressed in terms of vertical mode number.

This apparently seamless merger of the Poisson finescale world with the intrinsic internal wavefield is achieved subject to a significant constraint. The Poisson wavefield relation $\kappa_{0}^{-1}=2\left\langle\hat{\eta}^{2}\right\rangle / Z_{\text {corr_sL }}$ leads to

$$
\kappa_{0 \mathrm{GM}}=\left(\pi j_{*} b E\right)^{-1}=3 \cdot 9 / j_{*} \mathrm{~m}^{-1} \text {. }
$$

Typically, the bandwidth parameter $j_{*}$ is determined by matching observed vertical coherences of velocity or displacement with the GM model prediction. For the open-ocean datasets presented here, including Mixed Layer Dynamics Experiment (MILDEX), Patch Experiment (PATCHEX), and HOME Farfield, $\kappa_{0}=1-1.3$, consistent with $j_{*}=3-4$. However, $j_{*}$ is now more than just a fitting parameter. Combined with $E, j_{*}$ plays a critical role in establishing the non-Gaussian nature of the thermocline in the Poisson subrange $\kappa_{0}^{-1}<\overline{\Delta z}<Z_{\text {corr_sL }}$.

The displacement spectrum [Eq. (13)] can be multiplied by $(2 \pi k)^{2}$ to form an associated spectrum of vertical strain, $\gamma=$ $\partial \eta / \partial z$. Polzin (1995), Kunze (2017), and others have found the level of the strain spectrum to be a useful metric for modeling ocean mixing rates. Interestingly, the GM strain spectrum has magnitude $\kappa_{0}^{-1}$ throughout the Poisson subrange [Eq. (13a),
Fig. 3a, bottom panels], independent of all possible variations in $E, j_{*}, N(z)$, or $b$, provided the Poisson wavefield relation is maintained.

With a white strain spectrum, the GM strain variance increases without bound as smaller scales are considered. Munk (1981), proposed that the spectrum (13) be cut off at some limiting wavenumber $k_{u}$ and that a $k^{-1}$ spectral form be fitted to the strain spectrum $\left(k^{-3}\right.$ in displacement $)$, extending from $k_{u}$ to $1 \mathrm{cpm}$. Consistency with observations led to the selection of the strain (and shear) spectral slope. Consistency also required $k_{u}=1 / 10 \mathrm{cpm}$, independent of $N(z)$. This conflicts with the behavior of linear waves under WKB scaling. The determination of $k_{u}$ plays a huge role in the GM model's allocation of wavefield variance (see appendix in Gregg and Kunze 1991). Approximately 2/3 of the shear and strain variance in the GM spectrum lies in the $k_{u}<k<1 \mathrm{cpm}$ regime.

Munk (1981) explored various parameters of nonlinearity, referring to $k_{u}$ as the compliant wave cutoff and suggesting that $k_{u}$ varies inversely with wave energy, such that a universal value of $E k_{u}$ exists. In subsequent observations of shear (e.g., Müller et al. 1992) and strain (Fig. 6 in Part I), this appears to hold. The proper prediction of the relationship between $E$ and $k_{u}$ is considered a test of the veracity of dynamical models (e.g., Allen and Joseph 1989; Chunchuzov 1996, 2002; Lvov et al. 2004).

At high wavenumber, $k>k_{u}$, can the Poisson model wavenumber spectrum $S_{\gamma}^{L}(k)$, [Part I, Eq. (3)], be merged with the GM spectrum [Munk 1981; Eq. (13) here] while also maintaining Munk's hypothesis that the product $E k_{u}$ is universal? This is potentially challenging given that the Poisson spectrum is based on the statistical behavior of a stack of conceptual Poisson elements. But the low-wavenumber form of the Poisson strain spectrum is white and of level $\kappa_{0}^{-1}$, exactly matching the high-wavenumber level of the GM strain spectrum, provided the Poisson wavefield relation is maintained. The Poisson spectrum exhibits a cutoff at scale $k_{u}^{-1} \sim 2 \pi / \kappa_{0}=$ $6-10 \mathrm{~m}$ in an isopycnal-following frame, approximating Munk's $k^{-1}$ cutoff. In an Eulerian frame the Poisson strain spectrum cuts off at a slightly larger scale (Fig. 5 in Part I). Furthermore, the Poisson wavefield relation requires that $Z_{\text {corr_sL }} / \kappa_{0}$ vary with wavefield energy, $E \sim 2\left\langle\hat{\eta}^{2}\right\rangle$. Munk's $k_{u}$ will indeed vary inversely with energy, but only if the vertical correlation scale of the wavefield remains fixed.

This modification to Munk's hypothesis is significant, but also in line with observational experience. As a thought experiment, one can take a typical open-ocean wavefield (e.g., PATCHEX) and add a very energetic mode-1 internal tide (e.g., HOME Farfield) that interacts minimally with the broadband wavefield. The vertical correlation scale $Z_{\text {corr_sL }}$ and wavefield potential energy will both increase relative to the nontidal site, but $k_{u}, \kappa_{0}$, and the non-Gaussian nature of the thermocline will be minimally altered. Alternatively, one can uniformly increase the overall level of the internal wave spectrum, keeping $Z_{\text {corr_sL }}$ fixed. Here wavefield energy and $k_{u}, \kappa_{0}$ vary inversely, in accord with Munk's hypothesis.

Given that both the Poisson and GM spectral levels track perfectly, one can impose a high wavenumber cutoff on the 

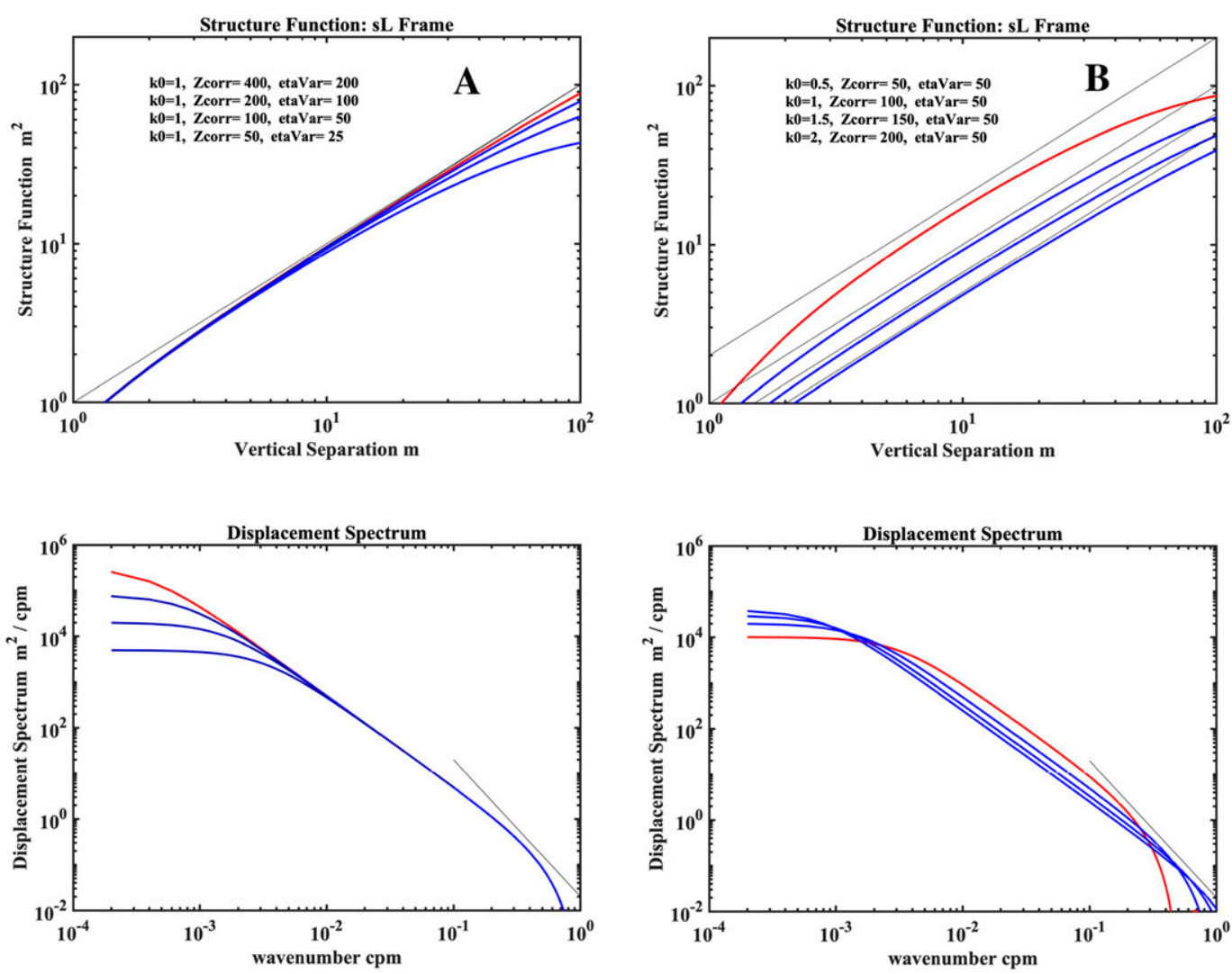

FIG. 3. The GM_P structure function and displacement spectrum. (a) The GM_P displacement variance can change by a factor of 8 , with no change in structure function, spectral level, or predicted dissipation rate, provided $\kappa_{0}$ is held constant. (b) Conversely, by varying $\kappa_{0}$ rather than displacement variance, changes are seen at all scales in the structure function and displacement spectrum. The black reference represents a $k^{-3}$ wavenumber dependence, that of the Munk (1981) version of the GM spectrum at scales smaller than $10 \mathrm{~m}$.

GM spectrum simply by multiplying the GM displacement or strain spectrum by $\kappa_{0} S_{\gamma \mathrm{SL}}(k)$ [Eq. (3) in Part I]. The resulting semi-Lagrangian (sL) GM_Poisson (GM_P) spectrum is

$$
\begin{aligned}
S_{\eta \mathrm{GM}_{-} \mathrm{P}}(k)= & \kappa_{0}^{-1}\left\{\frac{1}{\left[\left(2\left\langle\hat{\eta}^{2}\right\rangle \kappa_{0}\right)^{-2}+(2 \pi k)^{2}\right]}\right\} \sin c^{2}\left(k / \kappa_{0}\right), \\
& -\infty<k<\infty,
\end{aligned}
$$

$S_{\eta \mathrm{GM}_{-} \mathrm{P}}(k)=\frac{2\left\langle\hat{\eta}^{2}\right\rangle}{Z_{\text {corr_sL }}}\left\{\frac{1}{\left[Z_{\text {corr_sL }}^{-2}+(2 \pi k)^{2}\right]}\right\} \sin ^{2}\left(2 k\left\langle\hat{\eta}^{2}\right\rangle / Z_{\text {corr_sL }}\right)$,

$$
S_{\eta \mathrm{GM}_{-} \mathrm{P}}(k)=\frac{b^{3} E N_{0}^{2}}{j_{*} \pi N(z)^{2}}\left\{\frac{\beta_{*}^{2}}{\left[\beta_{*}^{2}+(2 \pi k)^{2}\right]}\right\} \sin c^{2}\left(\pi k j_{*} b E\right) \text {. }
$$

Here, (17a) gives the two-sided GM_P displacement spectrum in terms of Poisson variables, while (17b) makes use of (10) to present the spectrum in terms of intrinsic wavefield variables and (17c) uses GM variables.
Regarding the observational requirement that $k_{u}$ be independent of depth, in violation of the WKB approximation, Eq. (10) gives $\kappa_{0}$ as the ratio of $Z_{\text {corr_sL }}$ to $2\left\langle\hat{\eta}^{2}\right\rangle$. These outer scale quantities both vary as $N_{0} / N(z)$ in accord with WKB scaling. Thus $\kappa_{0}$ and the associated spectral cutoff $k_{u}=\kappa_{0} / 2 \pi$ is depth independent.

The associated GM_P vertical wavenumber spectrum for strain, $S_{\gamma \mathrm{GM} \_\mathrm{P}}(k)$ is again related to the displacement spectrum $S_{\eta \mathrm{GM} \_\mathrm{P}}(k)$ by the factor $(2 \pi k)^{2}$. Note that no matter how the parameters of the GM model are varied, the GM_P strain variance is very close to unity, provided the Poisson wavefield relation (10) is maintained.

The GM_P model covariance function $R_{\eta \mathrm{GM}_{\mathrm{P}} \mathrm{P}}(\overline{\Delta z})$ and structure function $M_{\eta \mathrm{GM}}^{2}(\overline{\Delta z})$ are of interest in many applications. The covariance is generated by convolving the two-sided GM covariance $R_{\eta \mathrm{GM}}(\overline{\Delta z})$ (12) and the Poisson covariance $R_{\gamma}^{L}(\overline{\Delta z})$ [Part I, Eq. (2)]. At separations $\kappa_{0}|\overline{\Delta z}| \ll Z_{\text {corr_sL }}$,

$$
R_{\eta \mathrm{GM}}(\overline{\Delta z}) \approx\left\langle\hat{\eta}^{2}\right\rangle\left(1-|\overline{\Delta z}| / Z_{\text {corr_sL }}\right)=\left\langle\hat{\eta}^{2}\right\rangle\left(1-\beta_{*}|\overline{\Delta z}|\right) .
$$

Given that $R_{\gamma}^{L}(\overline{\Delta z})$ is also triangular, the convolution can be performed analytically: 
$R_{\eta \mathrm{GM}_{\mathrm{P}} \mathrm{P}}(\overline{\Delta z})=\left\langle\hat{\eta}^{2}\right\rangle-\frac{1}{6 \kappa_{0}^{2}}-\frac{\overline{\Delta z}^{2}}{2}+\frac{\kappa_{0}|\overline{\Delta z}|^{3}}{6}+\cdots, \quad 0 \leq \kappa_{0}|\overline{\Delta z}| \leq 1$,

$R_{\eta \mathrm{GM}_{-} \mathrm{P}}(\overline{\Delta z})=\left\langle\hat{\eta}^{2}\right\rangle e^{-|\overline{\Delta z}| / Z_{\text {cor_ssL }}}, \quad \kappa_{0}|\overline{\Delta z}| \geq 1$.

The convolution rounds off the covariance peak at $\overline{\Delta z}=0$, reducing GM_P displacement variance by $1 /\left(6 \kappa_{0}^{2}\right)$. This reduction results from imposing the cutoff on the displacement spectrum at $\kappa_{u}=\kappa_{0} /(2 \pi) \mathrm{cpm}$.

The curvature of the GM_P covariance at $\overline{\Delta z}=0$ is a measure of the ratio of strain to displacement variance,

$$
R_{\gamma \mathrm{GM} \_\mathrm{P}}(\overline{\Delta z})=-R_{\eta \mathrm{GM} \_\mathrm{P}}^{\prime \prime}(\overline{\Delta z})=1-\kappa_{0}|\overline{\Delta z}|, \quad 0 \leq \kappa_{0}|\overline{\Delta z}| \leq 1
$$

demonstrating the consistency between Eq. (2) in Part I and Eq. (20) here.

The inverse of this ratio defines the Taylor microscale, an important metric in turbulence theory. For internal waves,

$$
\lambda^{2}=-\frac{2 R_{\eta \mathrm{GM} \_\mathrm{P}}(0)}{R_{\eta \mathrm{GM} \_\mathrm{P}}^{\prime \prime}(0)}=2\left\langle\hat{\eta}^{2}\right\rangle=Z_{\text {corr_sL }} / \kappa_{0} .
$$

In a Poisson thermocline, $\lambda$ is far from micro. Given that strain variance is always unity in the GM_P model, it is not surprising that the Taylor microscale is a metric of displacement variance.

In terms of the structure function of displacement, $M_{\eta}^{2}(\overline{\Delta z})$, the GM_P correlation function (19a) implies

$$
\begin{aligned}
M_{\eta \mathrm{GM}_{-} \mathrm{P}}^{2}(\overline{\Delta z}) & =2\left\{\left[\left\langle\hat{\eta}^{2}\right\rangle-1 /\left(6 \kappa_{0}^{2}\right)\right]-R_{\eta \mathrm{GM}_{-} \mathrm{P}}(\overline{\Delta z})\right\} \\
& =\overline{\Delta z}^{2}-1 / 3 \kappa_{0}|\overline{\Delta z}|^{3} .
\end{aligned}
$$

This result, derived from the convolution of second-order descriptors $R_{\gamma}^{L}(\overline{\Delta z})$ and $R_{\eta P} \approx R_{\eta \mathrm{GM}}$, is identical to Poisson microscale result (appendix C, section a in Part I), derived from the behavior of a vertical stack of hypothetical Poisson elements, with thickness governed by the exponential PDF.

The behavior of the GM_P structure function and displacement spectrum is illustrated in Fig. $3 \mathrm{a}$ as $\left\langle\hat{\eta}^{2}\right\rangle$ varies with $\kappa_{0}$ held constant, and in Fig. $3 \mathrm{~b}$ at fixed $\left\langle\hat{\eta}^{2}\right\rangle$ under variations in $\kappa_{0}$. At fixed $\kappa_{0}$, the GM_P structure function is insensitive to variations in both $\left\langle\hat{\eta}^{2}\right\rangle$ and $N^{2}(z)$ at separations $\Delta z<Z_{\text {corr_sL. }}$. The associated wavenumber spectral level also remains fixed for $k>\beta_{*} / 2 \pi=1 /\left(2 \pi Z_{\text {corr_sL }}\right)$, with changes in displacement variance occurring at smaller wavenumbers. The dependence of the displacement and strain spectra on $N^{2}(z)$ appears through changes in $\beta_{*}=1 / Z_{\text {corr_sL }}$.

The mathematical forms of the GM_P strain and displacement spectra are essentially unchanged from the Munk (1981) version of the GM model. However, there is now the implication that skewness and other moments of the strain field can be determined at all vertical scales from outer scale quantities like spectral level. Also, the low-wavenumber spectral bandwidth $\beta_{*}=1 / Z_{\text {corr_sL }}$ is now linked to the high wavenumber spectral cutoff $k_{u}=1 /\left(2 \pi \kappa_{0}\right)$ through the Poisson wavefield relation. These conceptual advances are associated with the underlying Poisson structure of the thermocline.

\section{Ocean turbulence and the Poisson subrange}

Through the Poisson wavefield relation, the Poisson constant $\kappa_{0}$ is seen to play many roles, including setting the level of the internal wave spectrum. The appearance of this meter-scale, non-Gaussian parameter in an internal-wave-scale spectral model makes sense in the context of a cross-scale energy cascade where the Poisson deformation of the thermocline plays an integral role. Small-scale turbulent dissipation must lie at the end of the cascade, somehow coexisting with a Poisson microscale that is here based on the concept of reversible fine structure, an adiabatic concept. The challenge is to link these seemingly disparate processes.

In the ocean, a defining property of turbulent mixing is its intermittency. Active overturning occurs between two (midgyre thermocline sites) and 30 (near-seafloor tidal conversion sites) percent of the time. When a turbulent event develops, is it aware of the preexisting steppiness of the thermocline? A growing convective instability developing on a small-scale wave might expand vertically through the low-gradient layer (wave crest) with further expansion inhibited by bounding high gradient sheets. The pioneering simulations of Orlanski and Bryan (1969) illustrate this process. Alternatively, a growing Kelvin-Helmholtz instability, perhaps originating on a sheet, might expand until limited by adjacent sheets. ${ }^{3}$

To establish a link between Poisson-scale and turbulent processes, one can compare the correlation scale $\left(\sim \kappa_{0}^{-1}\right)$ of irregularities in the reversible thermocline with the Thorpe (Th; Thorpe 1977) or Ozmidov (Oz) scales observed during periods of active turbulence. Here the Ozmidov scale is given by $\mathrm{Oz}=\sqrt{\epsilon / N^{3}}=0.8 \mathrm{Th}$, and the relationship to the Thorpe scale is based on the pioneering observations of Dillon (1982).

For the more recent cruises, a routine developed by J. Klymak (2016, personal communication), incorporating the Galbraith and Kelly (1996) overturn quality metric as well as other refinements, has been applied to the profiling datasets. Strain statistics obtained during periods of stable stratification can be compared with Thorpe scales derived from the imbedded turbulent events. ${ }^{4}$ A linear relationship is found between the

\footnotetext{
${ }^{3}$ The causal relationship between low-gradient regions in the thermocline and the occurrence of turbulence is a subject of longstanding debate. Stommel and Fedorov (1967), for example, conjectured that such layers represented the "scars" of preexisting patches of intense turbulence. In the present observations, where vertical density profiles are repeated rapidly in time, overturning is not seen to establish thermocline structure in abrupt events. Even in sites of massive $(\sim 50+\mathrm{m})$ convective overturns, the low-gradient crests develop and recede on internal wave time scales, with breaking abruptly occurring long after the local density gradient has begun to decrease.

${ }^{4}$ The profile segments from periods of active overturning can be Thorpe sorted, and "strain" can be calculated. The results are a slightly noisier version of the strain profiles that immediately precede and follow the event, given the short profile repeat intervals (4-15 min) employed here. Including these profiles along with the wave-strained profiles changes the overall averages very little.
} 


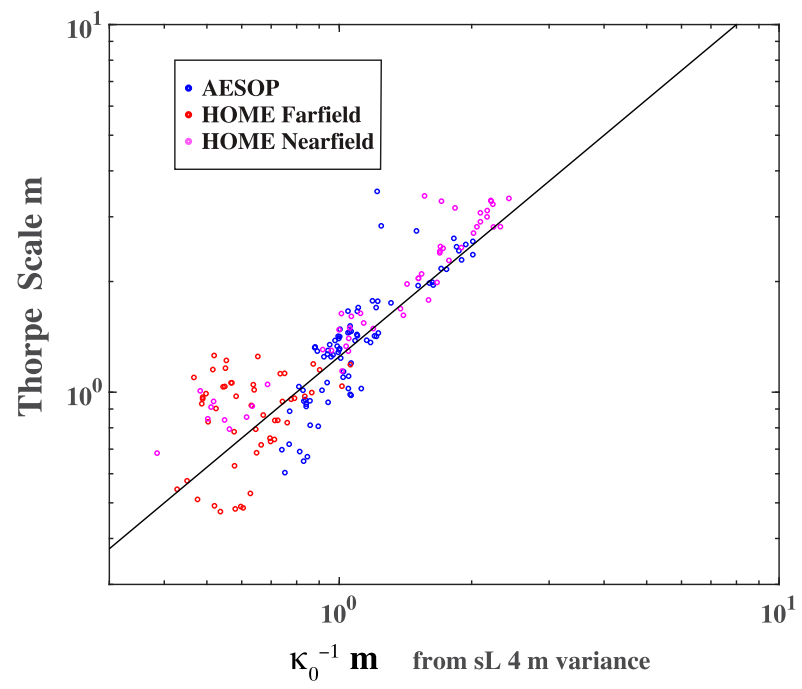

FIG. 4. A comparison of the observed Thorpe scale of overturning events with the inverse Poisson constant estimated from 4-m strain variance determined during periods when the stratification is stable. The individual points represent averages over $20-\mathrm{m}$ depth intervals from 100 to $800 \mathrm{~m}$ and over 1500 (AESOP) to 8100 (Nearfield) profiles (Part I, Table 1). Averages are formed in isopycnal coordinates. A similar pattern is seen in Eulerian frame averages. The reference line is $\mathrm{Th}=1.25 \kappa_{0}^{-1}$.

correlation scale $\kappa_{0}^{-1}$ of the reversible thermocline and the Thorpe scale (Fig. 4). Data from three sites are presented, with brief cruise summaries presented in Table 1 of Part I. The HOME Farfield represents a classic open-ocean site, with a GMlike wavefield complemented by an energetic mode- 1 semidiurnal tide. Vertical displacement variance scales in accord with the WKB approximation at this site. The Poisson constant $\kappa_{0}$ is nearly independent of depth $\overline{N^{2}}$, and is of order unity, as are the event Thorpe scales, averaged over the cruise duration and 20-m vertical intervals. Approximately $3000 \mathrm{~km}$ of CTD downcast data cluster in a small range of $\mathrm{Th}-\kappa_{0}^{-1}$ space.

The 2006 Assessing the Effects of Submesoscale Ocean Parameterizations (AESOP) cruise was sited in 1100-m water off the coast of Monterey, California. The lowest modes of an open-ocean wavefield are not present at this coastal site. Both $\left\langle\hat{\eta}^{2}\right\rangle$ and $Z_{\text {corr_sL }}$ are smaller than in the Farfield. There is weak tidal generation as well as reflection from the irregular seafloor. Strain variance increases, as does $\kappa_{0}^{-1}$, in the $400 \mathrm{~m}$ above the sea floor. Th mirrors this pattern linearly, with a constant of proportionality near unity. Approximately $1300 \mathrm{~km}$ of downcast data are applied to this result.

The HOME Nearfield Experiment took place atop Kaena Ridge, Hawaii, at 1100-m depth in a surrounding 4800-m ocean. The Ridge is a site of strong barotropic to baroclinic conversion. The upper $800 \mathrm{~m}$ of the water column were sampled, with strain variance and $\kappa_{0}^{-1}$ increasing near the bottom of the observation window. Corresponding cruise-average values of Th increase in step. Approximately $6000 \mathrm{~km}$ of CTD downcast data comprise the Nearfield contribution.

In preparing Fig. 4, it was necessary to specify a threshold on the Thorpe scale, to discriminate between events and nonevents. A threshold of $0.1 \mathrm{~m}$ is used here and in Fig. 5. The smaller values of average $\mathrm{Th}$ are sensitive to this choice of threshold. Similarly, an assumed strain noise variance of 0.125 in AESOP and Farfield, 0.375 in the Nearfield is subtracted from the observed strain variance before estimates of $\kappa_{0}$ are formed. This corresponds to an uncertainty in estimating the depths of individual isopycnals of $1 \mathrm{~m}$ (and $1.7 \mathrm{~m}$ in the HOME Nearfield). This correction weakly affects the constant of proportionality between Th and $\kappa_{0}^{-1}$. The high correlation is not altered.

The robustness of this result is not sensitive to the use of " $4-\mathrm{m}$ strain" to estimate $\kappa_{0}$, as opposed to estimates made at some other vertical scale. In Figs. 1 and A1, it is seen that a measurement of strain variance or mean cube strain anywhere within the Poisson subrange $\kappa_{0}^{-1}<\overline{\Delta z}<Z_{\text {corr_sL }}$ leads to an equivalent estimate of $\kappa_{0}$.

It is of value to inquire whether this Poisson-Ozmidov relationship emerges only in long-term averages of wave and turbulent patch statistics in the thermocline or whether it is maintained on a day-to-day basis. If the latter, is it because the short-term variability in the wavefield is very small, reflecting a residence time for energy of weeks to months? Or do Th and $\kappa_{0}^{-1}$ vary in step on much shorter time scales?

In the 2015 TTIDE experiment, eight sites on the east coast of Tasmania were visited, each for a period of 1-2 days. A remotely generated baroclinic tide shoals and reflects on this coast, leading to extreme variability in the near-seafloor wave and turbulent fields. With an acoustic altimeter on the profiling CTD, the density field was monitored to within $15 \mathrm{~m}$ of the sea floor. Profiles could be repeated at 8-15-min intervals, slightly faster than the buoyancy period at the observed depths. Collectively, the observations spanned depths 10-2000 m. While these single-site datasets are a factor of 10-30 smaller than the others reported here, the range of variability in $\kappa_{0}$ and Thorpe scale in TTIDE is very large. Again, a strong correlation is seen between Th and $\kappa_{0}^{-1}$ in these short-term highly variable measurements (Fig. 5a) with a constant of proportionality near unity.

This observational link between the adiabatic Poisson constant and the scale of turbulent overturning has direct application to the modeling of ocean mixing. To progress, it is critical to maintain the distinction between reversible and irreversible processes. As a start, consider the event dissipation rate $\left\langle\varepsilon_{\text {event }}\right\rangle$ and the climatological mean dissipation rate $\langle\varepsilon\rangle$, as related by

$$
\langle\varepsilon\rangle=\left\langle\varepsilon_{\text {event }}\right\rangle \times \phi
$$

Here $\phi$ is an intermittency factor, the percentage of time that a nonzero Thorpe scale is detected on any given density surface. If we link $\kappa_{0}^{-1}$ to the event Ozmidov scale

$$
\kappa_{0}^{-1}=c_{0}\left(\left\langle\varepsilon_{\text {event }}\right\rangle /\left\langle N^{2}\right\rangle^{3 / 2}\right)^{1 / 2},
$$

then

$$
\left\langle\varepsilon_{\text {event }}\right\rangle=c_{0}^{-2}{\overline{N^{2}}}^{3 / 2} / \kappa_{0}^{2} .
$$

Equations (24) and (25) define the Poisson-Ozmidov relation. Here $c_{0}$ is a dimensionless constant of order unity. If $\kappa_{0}$ is independent of $\left\langle N^{2}\right\rangle$ in the open-ocean thermocline 


\section{1/Kappa0 vs Thorpe Scale Dn Profiles}

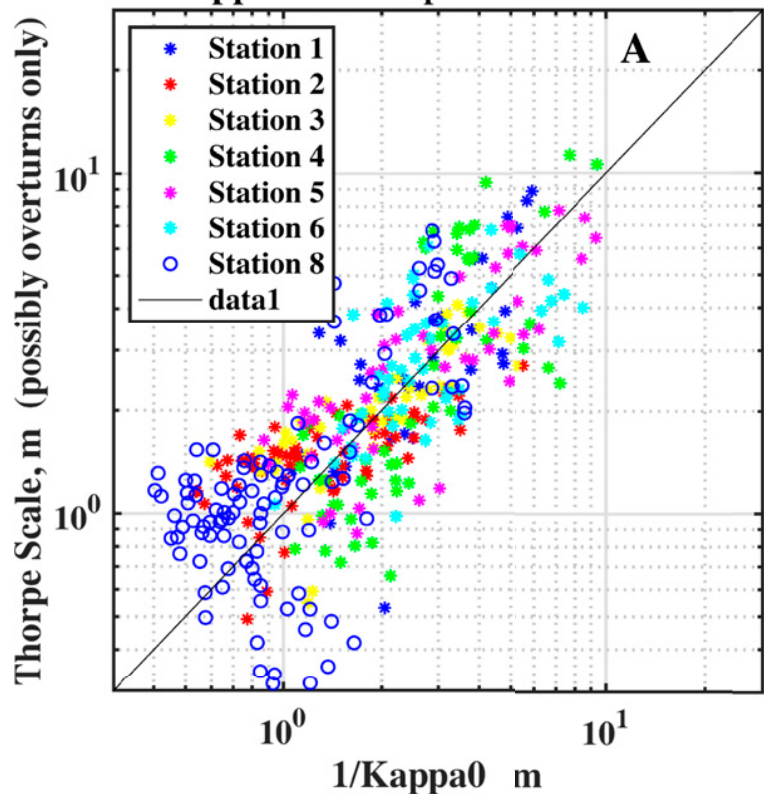

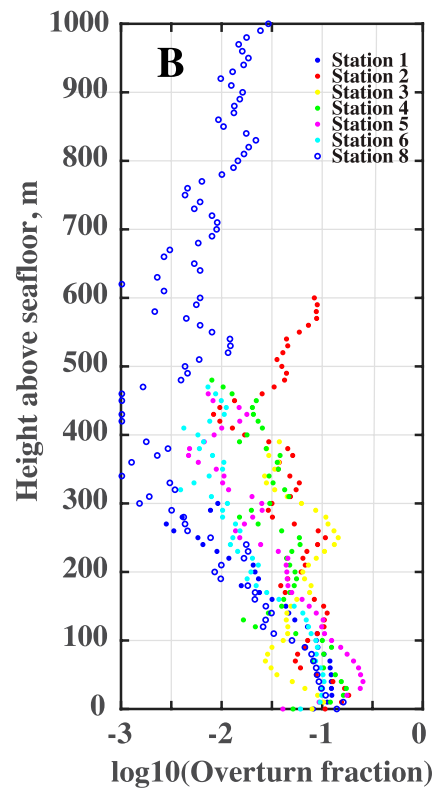

FIG. 5. In the 2015 TTIDE experiment, eight sites were occupied for 1-3 days. (a) A strong correlation is seen between $\kappa_{0}$ and the Thorpe scale of observed mixing events. An energetic baroclinic tide shoals and reflects at the TTIDE site. (b),(c) Both the fractional duration of overturning $\phi$ and the climatological mixing rate $\langle\varepsilon\rangle$ increase as the seafloor is approached, in contrast to classical open-ocean behavior, where $\langle\varepsilon\rangle \sim \overline{N^{2}}$.

(Part I, Fig. 9), then the event dissipation rate varies as $\left\langle N^{2}\right\rangle^{3 / 2}$.

It is reasonable to assume that the intermittency factor $\phi$ is given by some ratio of the triggering time scale for turbulent events and the individual event duration. Examples might be

$$
\phi=c_{1} f / N(z) \text { or } \phi=c_{1} \mathrm{M}_{2} / N(z), \text { etc. }
$$

For these trial definitions, the corresponding climatological dissipation rate varies as

$$
\langle\varepsilon\rangle \sim \overline{N^{2}} \text { and } K_{\rho} \sim\left(\overline{N^{2}}\right)^{0}
$$

consistent with most open-ocean observations. Here $f$ is the local inertial frequency, $\mathrm{M}_{2}$ is the frequency of the semidiurnal tide, and $c_{1}$ is a dimensionless constant. Noting the $\kappa_{0}^{-2}$ dependence in $\varepsilon(25)$, we can invoke the Poisson wavefield relation to see

$$
\begin{aligned}
& \langle\epsilon\rangle_{\text {IW_P }}=c_{1} c_{0}^{-2} f \overline{N^{2}} / \kappa_{0}^{2}, \\
& \langle\epsilon\rangle_{\text {IW_P }}=4 c_{1} c_{0}^{-2} f \overline{N^{2}}\left\langle\eta^{2}\right\rangle^{2} / Z_{\text {corr_sL }}^{2}, \\
& \langle\epsilon\rangle_{\text {IW_P }}=c_{1} c_{0}^{-2} f \overline{N^{2}} \pi^{2} b^{2} E^{2}=c_{1} c_{0}^{-2} f N_{0}^{2} E_{0}^{2} \pi^{2} b^{2}\left(\overline{N^{2}} / N_{o}^{2}\right)\left(E^{2} / E_{0}^{2}\right) .
\end{aligned}
$$

Here we have set $\phi=c_{1} f / N$ and presented the result in terms of Poisson scale (28a), internal wave scale (28b), and GM parameters (28c), as expressed in (14) and (15).

If the constants $c_{0}$ and $c_{1}$ are set to unity and $\langle\varepsilon\rangle_{\text {IW_P }}$ is evaluated at $30^{\circ}$ latitude and at the Garrett-Munk energy level, we find

$$
\begin{aligned}
& \langle\epsilon\rangle_{\mathrm{IW}_{-} \mathrm{P}}=f N_{0}^{2} \times\left(\pi b j_{*} E\right)^{2}=4.37 \times 10^{-11} j_{*}^{2}, \\
& \langle\epsilon\rangle_{\mathrm{IW} \_\mathrm{P}}=3.9 \times 10^{-10} \mathrm{~W} \mathrm{~kg}^{-1}, \text { for } j_{*}=3, \\
& \langle\epsilon\rangle_{\mathrm{IW} \_\mathrm{P}}=7 \times 10^{-10} \mathrm{~W} \mathrm{~kg}^{-1}, \quad \text { for } \quad j_{*}=4 .
\end{aligned}
$$

These compare with $\langle\epsilon\rangle_{\text {IW_Gregg }}=7 \times 10^{-10} \mathrm{~W} \mathrm{~kg}^{-1}$ and $\langle\epsilon\rangle_{\text {IW_Polzin }}=8 \times 10^{-10} \mathrm{~W} \mathrm{~kg}^{-1}$ from the Gregg 1989 synthesis of data and theory and the more recent study by Polzin et al. (2014).

Equation (28a) is the Poisson version of the Gregg-Henyey relation. The original Gregg-Henyey relation has been modified over the years (e.g., Polzin et al. 1995; Hibiya et al. 2012; Polzin et al. 2014) to include adjustments for both latitude and shear-to-strain ratio. These data from HOME Nearfield, AESOP, and TTIDE demonstrate the validity of the PoissonOzmidov relation (24) (Figs. 4 and 5a), even in the absence of a continuous energy cascade through the internal wave spectrum. In these regions of intensified near-seafloor mixing, the variability of $\kappa_{0}$ and $\langle\varepsilon\rangle$ with $\overline{N^{2}}$ differs significantly from openocean behavior. This is reflected in the observed profiles of $\phi(z)$ (Figs. 5b and 5c), not in the relationship between Th and $\kappa_{0}$. One can conjecture that the Poisson-Ozmidov relation (24) remains valid universally and that site-dependent environmental and wavefield forcing factors primarily influence intermittancy.

\section{Discussion}

A Poisson subrange governed by the single parameter $\kappa_{0}$ is consistent with existing understanding of the larger-scale intrinsic internal wavefield and with the established parameterization of ocean turbulence. Given the linkage established 
between $\kappa_{0}$ and the vertical wavenumber spectrum of strain, global maps of non-Gaussian parameters such as skewness can now be produced for any vertical scale $\overline{\Delta z}$ in the Poisson subrange, based on the existing maps of strain variance that have been developed (e.g., Whalen et al. 2012; Kunze 2017) to evaluate the Gregg-Henyey model.

The Poisson constant $\kappa_{0}$, the metric for deformation, generally varies inversely with wavefield energy. But it is also linked with the vertical correlation scale of the wavefield $Z_{\text {corr_sL }}$ as specified by the Poisson wavefield relation [inverse of Eq. (10)],

$$
\kappa_{0}=Z_{\text {corr_sL }} /\left(2\left\langle\hat{\eta}^{2}\right\rangle\right),
$$

the bridging relationship between the Poisson domain and that of the intrinsic internal wavefield. The Relation emphasizes that the non-Gaussian nature of the thermocline is dependent both on the energy in the intrinsic internal wavefield and the vertical wavenumber bandwidth across which this energy is distributed. The Relation is demonstrated in Fig. 2 here and in Fig. 6 in Part I, where vertical wavenumber spectra of strain and displacement are rescaled using values of $\kappa_{0}$ estimated from $8 \mathrm{~m}$ strain skewness. The nondimensionalized spectra are seen to coincide to within a factor of 2 over vertical scales $10-200 \mathrm{~m}$. In excess of $30000 \mathrm{~km}$ of downcast CTD profile data contribute to these results.

A $k^{-2}$ spectral form is known to describe the internal wave displacement spectrum over a range of scales $\beta_{*} / 2 \pi<k_{z}<k_{u}$, where $2 \pi \beta_{*}^{-1} \approx 1000 \mathrm{~m}$ and $k_{u}^{-1} \approx 10 \mathrm{~m}$. This form is mathematically problematic in that, if extended across all scales, both infinite displacement and infinite strain variance result. Taking the Munk (1981) version of the GM model to specify the lowwavenumber behavior of the spectrum, it is shown that a Poisson model of the high-wavenumber cutoff can be added, with the Poisson wavefield relation suggesting a number of surprising cross-scale linkages. Notably, the level of this hybrid GM_P spectrum is given by $\kappa_{0}^{-1}$ in the $k^{-2}$ spectral region. Also, if both displacement variance $\left\langle\hat{\eta}^{2}\right\rangle$ and the low-wavenumber cutoff $\beta_{*} \equiv 1 / Z_{\text {corr_sL }}$ scale in a WKB sense, the high-wavenumber cutoff $k_{u}=\kappa_{0} /(2 \pi)$ is independent of $N(z)$. These linkages make sense in the context of an energy cascade.

With more recent observations (HOME, AESOP, TTIDE), it is seen that $\kappa_{0}^{-1}$ is proportional to the observed Thorpe scale associated with density overturning events. These $\kappa_{0}$ estimates are based on the $70 \%-95 \%$ of the observations occurring when overturning is not present. The Thorpe scale estimates are averaged only over the overturning events. Given this Poisson-Ozmidov relation, it is straightforward to recast the Gregg-Henyey mixing model in terms of the Poisson formalism (28), leading to an expression for the GM dimensionless energy parameter

$$
E=\left(\pi j_{*} b \kappa_{0}\right)^{-1}=\left(\pi j_{*} b\right)^{-1}\left[\langle\epsilon\rangle_{\mathrm{IW}_{-} \mathrm{P}} /\left(f \overline{N^{2}}\right)\right]^{1 / 2} .
$$

In contemporary implementations of the Gregg-Henyey parameterization, the original formula has been modified to account for variations in both latitude and shear-to-strain variance ratio, representing the variability of the near-inertial peak relative to the GM norm. A modification that embraces strain to displacement ratio, a measure of the vertical wavenumber bandwidth of the wavefield, $Z_{\text {corr_sL }}^{-1}$, is probably in order as well.
With existing data, it is possible to see whether these modifications relate to changes in the Poisson-Ozmidov relation [(24) and (25)] or in the fractional incidence of overturning $\phi$.

The GM_P restatement of the Gregg-Henyey model has $\left\langle\varepsilon_{\text {event }}\right\rangle$ varying with wavefield energy level and correlation scale, while $\phi$ remains strictly a function of the background ocean. This conflicts with common experience, in that overturning events occur more frequently at intense mixing sites. More reasonably, $\phi$ might be defined as the fractional time of existence of the $X \%$ most energetic overturns, the rare events that dominate a long-term average. Given that observations of $\left\langle\varepsilon_{\text {event }}\right\rangle$ tend to vary over orders of magnitude at a single site, it makes sense that the model should focus on the dominant overturns that establish the climatological average.

The present custom is to report the climatological average $\langle\varepsilon\rangle$, rather than $\left\langle\varepsilon_{\text {event }}\right\rangle$ and $\phi$ separately. Going forward, it is clear that both event-averaged dissipation rates and the intermittency of the mixing events have distinct stories to tell. It is critical that a useful definition of $\phi$ be agreed upon and that both $\phi$ and $\left\langle\varepsilon_{\text {event }}\right\rangle$ be reported in observational campaigns.

It is useful to present a phenomenological hypothesis for the energy cascade. At the largest scales, $\overline{\Delta z} \geq Z_{\text {corr_sL }}$, intrinsic internal waves propagate essentially linearly, affected by background profiles of shear and $\overline{\left\langle N^{2}\right\rangle}$ as well as Earth's rotation. As vertical scale decreases, the waves become increasingly sensitive to the horizontal velocity variability of their larger-scale brethren. Munk's compliant waves have horizontal phase speeds less than the rms particle velocity of the larger-scale intrinsic constituents of the wavefield, with boundary $k_{c} \sim N(z) / U_{\text {rms }}$.

Munk was hoping to identify $k_{c}$ with $k_{u}$, the 10 -m cutoff, but for reasonable values of $U_{\mathrm{rms}}$ and $N(z)$, the compliant wave boundary appeared to be at much larger scale, more like $60 \mathrm{~m}$ [Munk 1981, Eq. (9.30)]. Holloway (1980) also found that the wavefield becomes strongly nonlinear at scales below $\sim 40 \mathrm{~m}$. While there is no spectral signature of this boundary, the nonGaussian aspect of the motion field begins to emerge at this scale. In the present data, estimates of the third-order structure function $M_{\eta}^{3}(\overline{\Delta z})$ (Figs. 1b and A2) rise above measurement noise and become significant at scales smaller than $\kappa_{c}^{-1}$. The vertical separation $\overline{\Delta z}$ of the observed maximum of the thirdorder structure function $M_{\eta}^{3}(\overline{\Delta z})$ might be a viable observational metric of Munk's $\kappa_{c}^{-1}$.

Regarding the sequence of events associated with this process (Figs. 7d and $8 \mathrm{e}$ in Part I), suggest that the short-wave packets that locally dominate thermocline strain are ones whose intrinsic frequencies have become small, such that their signatures are effectively embedded in the local mean flow. At vertical scales smaller than $\kappa_{c}^{-1}$, vertical waveforms are distinctly nonsinusoidal (Fig. 2 in Part I), associated with the production of forced harmonics in vertical wavenumber. Much of the spectral variance between $k_{c}$ and $\kappa_{0}$ is associated with the nonsinusoidal waveforms of these waves, as is the nonGaussian (Poisson) structure of scalar profiles in the thermocline. The vertical extent of the isopycnal "layers" and the resulting 10-m cutoff at $k_{u}$ are associated with wave displacement amplitude, not vertical wavelength (Figs. 7d-f and $8 \mathrm{e}$ in Part I). This is consistent with the scale separation between $k_{c}^{-1}=60 \mathrm{~m}$ and $\kappa_{0}^{-1} \sim 1 \mathrm{~m}$, with $k_{u}^{-1} \sim 2 \pi \kappa_{0}^{-1} \approx 10 \mathrm{~m}$. 
The development of nonsinusoidal vertical waveforms can be seen in numerical simulations of a wave encountering a critical layer. Excellent examples are presented by Winters and D'Asaro (1994, their Figs. 3-5). As wave amplitude builds, the vertical waveform becomes highly steppy. The "sheets and layers" are short lived in the Winters and D'Asaro (1994) Eulerian-frame simulation because their small-scale wave is given a high frequency and this remains constant throughout the encounter.

In terms of spectral descriptions, there is a significant difference between the cascade of energy through the internal wavefield and the cascade associated with isotropic turbulence. Increasing the energy flux through a turbulent field drives velocity variability to progressively smaller scales and eventual destruction by molecular viscosity. Increasing the energy flux through the internal wave spectrum causes compliant waves to become nonsinusoidal at larger vertical scale. Thermocline steps become larger and the correlation scale of the strain field, $\kappa_{0}^{-1}$, grows. This behavior is demonstrated in the Poisson simulation in Fig. 4 of Part I. It is seen observationally in Fig. 10 of Part I where 2-m strain variance, directly proportional to $\kappa_{0}^{-1}$, is greatest at tide-topography interaction sites such as TTIDE and HOME Nearfield.

One of the puzzles associated with the Gregg-Henyey parameterization of ocean turbulence is that it appears to give accurate predictions at geographically localized energetic sites such as TTIDE, as well as in regions of spatial homogeneity. In localized sites, a weakly nonlinear cascade through the full internal wave spectrum does not have the spatial extent to occur. Perhaps the thermocline steppiness that sets strain spectral level is most sensitive to the end-state of the cascade, the extreme nonlinear distortion of compliant waves that are on the edge of breaking. The state of these waves and the corresponding mixing rate vary together. The relationship between strain spectral level and observed wave breaking remains fixed while both fluctuate on a more rapid/more local scale.

Figures 7 and 8 in Part I show the role of larger-scale nearinertial shear, as well as near-inertial and tidal strain (Sun and Kunze 1999) in modulating the propagation of short waves. When inertial shear inhibits the vertical propagation of a wave packet, local wave amplitude grows and an irregular thermocline potentially develops. If the packet's intrinsic frequency remains above $f$, it can resume propagation a quarter inertial period later, returning to a more sinusoidal waveform. The formation of these adiabatic irregularities in conjunction with a causal background shear (and strain) must play a key role in orchestrating the presence of overturning and resultant ocean mixing.

Recently, Kunze (2019) presented a differing view of the end stages of the energy cascade. Focusing on a stationary homogeneous spectral description, Kunze argues that a field of anisotropic stratified turbulence lies between the wavenumbers of the shortest internal waves and the climatological Ozmidov scale. Such a model is not inconsistent with the Poisson structure of the thermocline. It does contrast with the view presented here, which is based on observations of internal waves directly breaking (e.g., Alford and Pinkel 2000). A brief comparison of the two views is presented in appendix B.

As the vertical resolution of numerical models improves and small-scale internal waves and submesoscale motions are admitted to the motion fields, the Poisson nature of the thermocline should be reproduced. Realistic models of diapycnal diffusion will require knowledge of the co-occurrence of the various types of wave breaking and the strain and shear state of the evolving thermocline. Both observational and theoretical guidance is necessary here if we are to capitalize on ongoing computational progress.

Acknowledgments. The author thanks Eric Slater, Lloyd Green, Mike Goldin, Tony Aja, Chris Neely, Mai Bui, Tyler Hughen, San Nguyen, Jonathan Ladner, and Sara Goheen for their participation in the development of the instrument systems described here and for the operation of these systems at sea. The seagoing leadership of Captains DeWitt Efird and Tom Golfinos (R/P FLIP) and Tom Desjardins and Dave Murline (R/V Roger Revelle) is greatly appreciated. Mike Gregg, Matthew Alford, Jennifer MacKinnon, Andrew Lucas, and Gregory Wagner provided valuable comments on drafts of this work. Jody Klymak processed the HOME and AESOP CTD information used to estimate the Thorpe scales in Fig. 4. Support from the National Science Foundation and the Office of Naval Research is gratefully acknowledged.

\section{APPENDIX A}

\section{Structure Functions of Isopycnal Separation from all Cruises}

While oceanographers are most familiar with spectral descriptions of the motion field at internal wave scales, higherorder descriptors are necessary to describe the increasingly non-Gaussian nature of the motions at smaller vertical scales. The second order structure function (Fig. A1) is a useful bridge between spectral and non-Gaussian descriptors, and the third order structure function (Fig. A2) is an excellent metric of the Poisson nature of the compliant wavefield and its transition to the Gaussian intrinsic regime. Here, data from Fig. 1 are repeated, along with results from the corresponding results from the other five sites used in this analysis.

\section{APPENDIX B}

\section{Compliant Waves, Vortical Motions, and Anisotropic Turbulence}

Kunze (2019) presents a differing view of the end stages of the energy cascade. Focusing on a stationary homogeneous spectral description, the steady-state transfer of energy is tracked through various spectral subranges. Kunze argues that a field of anisotropic stratified turbulence lies between the wavenumbers of the shortest internal waves $(\sim 1 / 10 \mathrm{~m})$ and the climatological Ozmidov scale,

$$
L_{O}=\left[\langle\varepsilon\rangle /\left(\overline{N^{2}}\right)^{3 / 2}\right]^{1 / 2}
$$

At scales smaller than $L_{o}$, the motion field is asserted to be steady, isotropic turbulence. The distinction between "event" and "climatological" averages is absent in his steady-state model. 


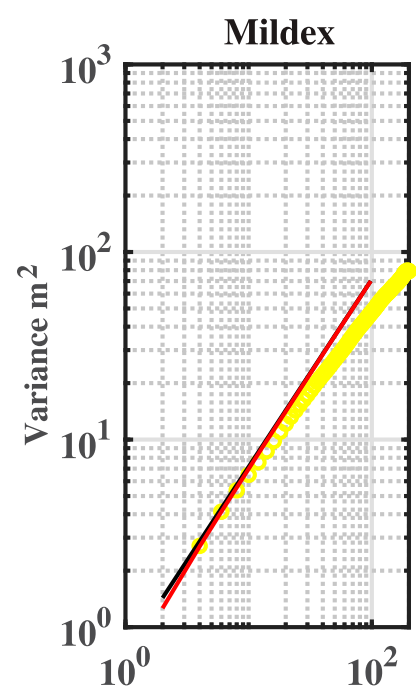

Mean separation $\mathbf{m}$

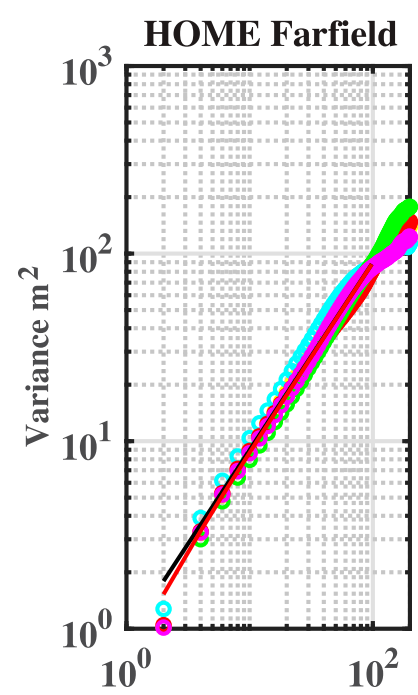

Mean separation m

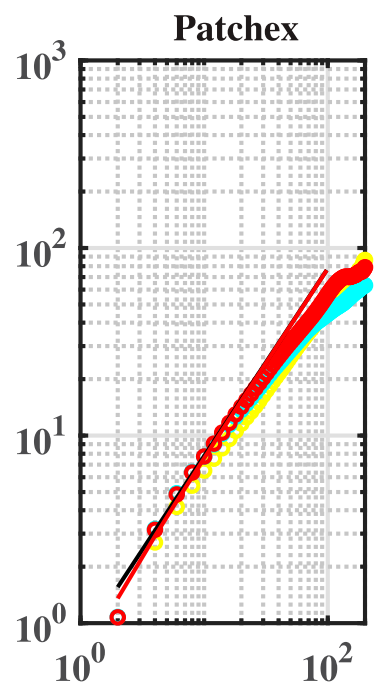

Mean separation $\mathbf{m}$

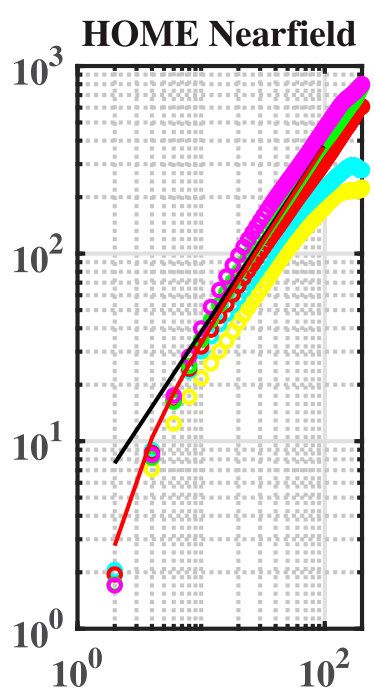

Mean separation $\mathbf{m}$

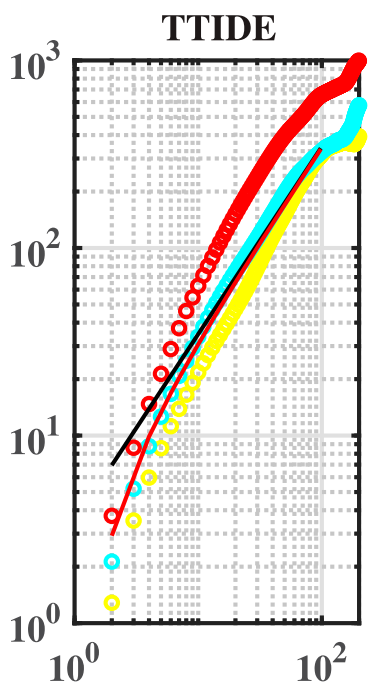

Mean separation $\mathbf{m}$

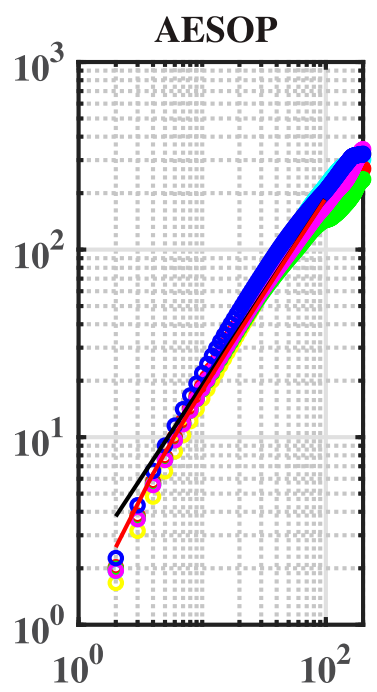

Mean separation m

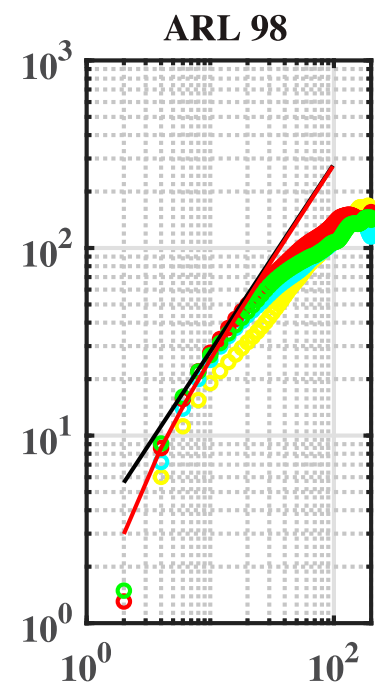

Mean separation $\mathbf{m}$

FIG. A1. Second-order structure functions of vertical displacement for the seven cruises. Reference lines give predictions based on the Poisson model (black) and the Poisson microscale model (red) (Part I, appendix B), using a single value of $\kappa_{0}$ derived from the PDF of 8-m strain (Part I, Fig. 3), averaged over all 200-m measurement intervals. For the open-ocean observations, MILDEX, PATCHEX, Farfield, and even the coastal AESOP data, the independence of the structure function with measurement depth $\bar{N}^{2}$ is striking. Sites of pronounced tidal generation (Nearfield) or dissipation (TTIDE) show significant increases in the structure function as the seafloor is approached.

It is useful to quantify representative values of the climatological Ozmidov scale. Consider an open-ocean thermocline with a climatological eddy diffusivity of order

$$
K_{\rho}=0.2\langle\varepsilon\rangle / N^{2} \sim 5.010^{-6} .
$$

The corresponding Ozmidov scale is

$$
L_{O}=\left[5 K_{\rho} /\left(\overline{N^{2}}\right)^{1 / 2}\right]^{1 / 2}
$$

For buoyancy frequencies of 1,3 , and $10 \mathrm{cph}$, associated values of $L_{O}$ are $0.12,0.07$, and $0.04 \mathrm{~m}$, much smaller than the height of the typical (intermittent) overturns seen in the thermocline. Increasing $K_{\rho}$ to $10^{-4}$ increases the corresponding $L_{0}$ by a factor of 4.5 , still a very small value.

Kunze's anisotropic turbulence at $\sim 0.1-10$-m vertical scales, is forced by short internal waves and it is this turbulence that ultimately breaks. Scaling arguments are used to infer the spectral forms of velocity and density variability in this subrange, with numerous plausible alternatives considered. This scaling-based approach goes beyond the present statistical exploration in that, with both $f$ and $N$ available as time scales, the velocity field can be considered as well as displacements and strains. 

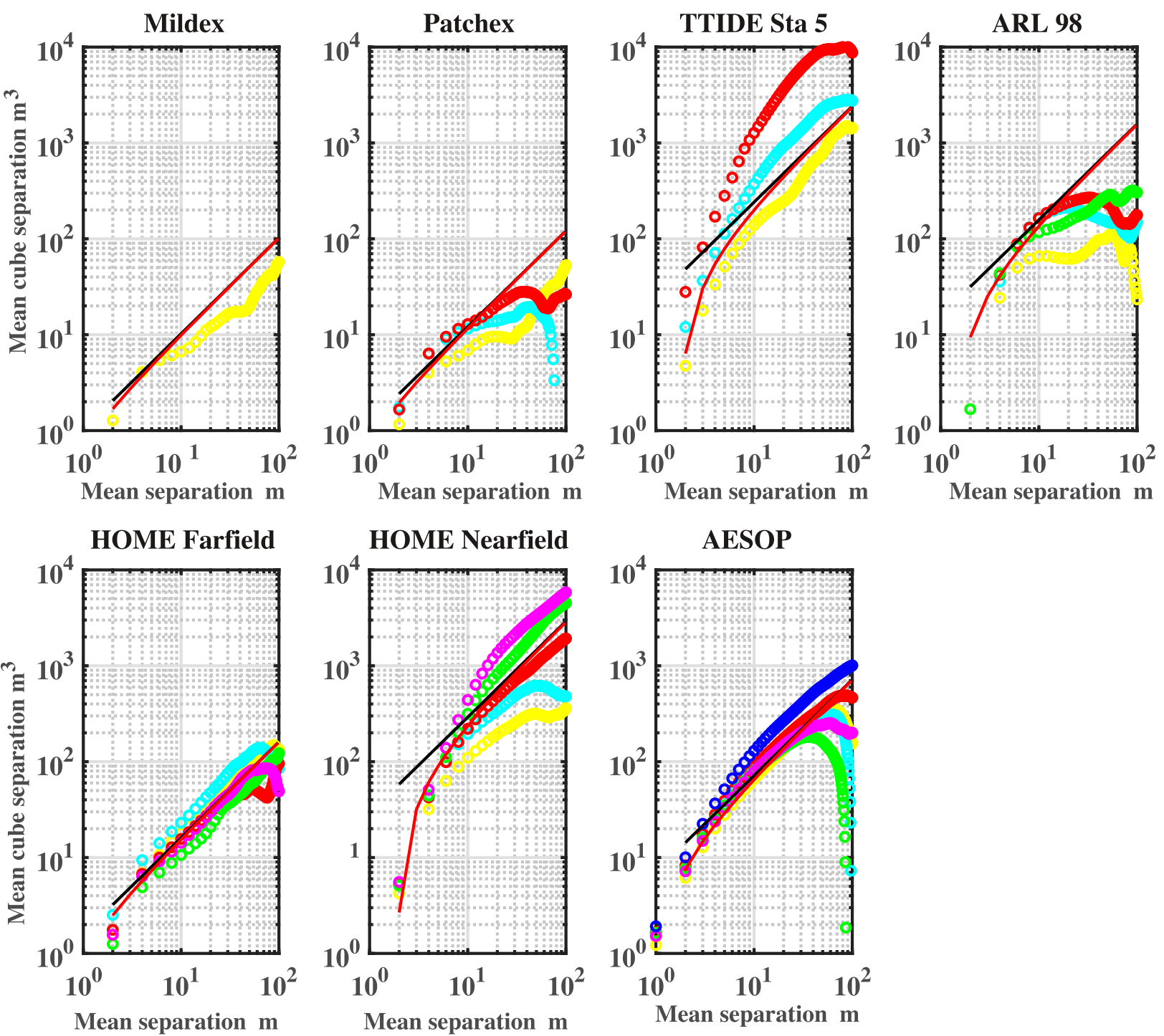

FIG. A2. Third-order structure functions of vertical displacement for the seven cruises. Reference lines give predictions based on the Poisson model (black) and the Poisson microscale model (red) (Part I, appendix B), using a single value of $\kappa_{0}$ derived from the PDF of 8-m strain (Part I, Fig. 3), averaged over all 200-m measurement intervals.

Vortical motions are indeed found at $0.1-10-\mathrm{m}$ vertical scales in the sea, and they can be distinguished from internal waves in wavenumber frequency spectra of strain, Pinkel (2014). Are these motions primarily forced by small scale waves, perhaps when a wave packet locally deposits momentum to the mean flow due to a critical layer encounter? A hallmark of the vortical signal seen in wavenumber frequency spectra is that vortical variance is found in a spectral ridge centered at zero frequency. The ridge is clearly broadened by Doppler shifting associated with lateral advection, but its subinertial origin is clear. In Figs. $7 \mathrm{~b}$ and $7 \mathrm{~d}$ of Part I, the strain field is presented both with and without the vortical ridge present. One might think that Kunze's wave-forced anisotropic turbulence is not strongly concentrated at subinertial frequencies. If so, it should be clearly apparent in Fig. 7d. Numerical simulations of a laterally as well as vertically localized wave packet encountering a critical layer will be instructive in addressing this concern.

The Poisson model presented here has only a single parameter available for adjustment. It leaves virtually no room for subjective choice in considering both spectral descriptions and higher-order statistics. The fact that the model seems equally applicable at energetic sites such as TTIDE and the HOME Nearfield, where the local baroclinic tide is breaking directly on topography, and at the HOME Farfield, PATCHEX, and MILDEX sites, where a classical open-ocean energy cascade is established, suggests that it is a robust descriptor of the non-Gaussian behavior of the thermocline. Thermocline irregularities formed by the nonsinusoidal vertical waveforms of nonlinear internal waves develop the same high aspect ratio, $k_{z} / k_{x}$, as does Kunze's stratified turbulence. In terms of 
wavenumber spectra, the spectral signatures might not be very different.

The dynamics of the high-wavenumber end of the energy cascade is best explored by observing systems that document the progression of events leading to intermittent mixing (e.g., Alford and Pinkel 2000).

\section{REFERENCES}

Alford, M. H., and R. Pinkel, 2000: Observations of overturning in the thermocline: The context of ocean mixing, Part I. J. Phys. Oceanogr., 30, 805-832, https://doi.org/10.1175/1520-0485(2000) 030<0805:OOOITT $>2.0$.CO;2.

Allen, K. R., and R. I. Joseph, 1989: A canonical statistical theory of oceanic internal waves. J. Fluid Mech., 204, 185-228, https:// doi.org/10.1017/S0022112089001722.

Chunchuzov, I. P., 1996: The spectrum of high-frequency internal waves in the atmospheric wave-guide. J. Atmos. Sci., 53, 1798-1814, https://doi.org/10.1175/1520-0469(1996)053<1798: TSOHFI $>2.0 . \mathrm{CO} ; 2$.

_- 2002: On the high-wavenumber form of the Eulerian internal wave spectrum in the atmosphere. J. Atmos. Sci., 59, 1753-1774, https://doi.org/10.1175/1520-0469(2002)059<1753: OTHWFO $>2.0 . \mathrm{CO} ; 2$.

Dillon, T. M., 1982: Vertical overturns: A comparison of Thorpe and Ozmidov length scales. J. Geophys. Res., 87, 9601-9613, https://doi.org/10.1029/JC087iC12p09601.

Galbraith, P. S., and D. E. Kelly, 1996: Identifying overturns in CTD profiles. J. Atmos. Oceanic Technol., 13, 688-702, https:// doi.org/10.1175/1520-0426(1996)013<0688:IOICP>2.0.CO;2.

Garrett, C. J. R., and W. H. Munk, 1972: Space-time scales of internal waves. Geophys. Fluid Dyn., 3, 225-264, https://doi.org/ 10.1080/03091927208236082.

- , and - , 1975: Space-time scales of internal waves: A progress report. J. Geophys. Res., 80, 291-297, https://doi.org/ 10.1029/JC080i003p00291.

Gregg, M., 1989: Scaling turbulent dissipation in the thermocline. J. Geophys. Res., 94, 9686-9698, https://doi.org/ 10.1029/JC094iC07p09686.

Gregg, M. C., and E. Kunze, 1991: Shear and strain in Santa Monica basin. J. Geophys. Res., 96, 16709-16719, https://doi.org/ 10.1029/91JC01385.

Henyey, F. S., and J. Wright, and S. M. Flatté, 1986: Energy and action flow through the internal wave field: An Eikonal approach. J. Geophys. Res., 91, 8487, https://doi.org/10.1029/ JC091iC07p08487.

Hibiya, T., N. Furuichi, and R. Robertson, 2012: Assessment of fine-scale parameterizations of turbulent dissipation rates near mixing hotspots in the deep ocean. Geophys. Res. Lett., 39, L24601, https://doi.org/10.1029/2012GL054068.

Holloway, G., 1980: Oceanic internal waves are not weak waves. J. Phys. Oceanogr., 10, 906-914, https://doi.org/10.1175/ 1520-0485(1980)010<0906:OIWANW $>2.0 . \mathrm{CO} ; 2$.

Kunze, E., 2017: Internal-wave-driven mixing: Global geography and budgets. J. Phys. Oceanogr., 47, 1325-1345, https:// doi.org/10.1175/JPO-D-16-0141.1.

__ 2019: A unified model for anisotropic stratified and isotropic turbulence in the ocean and atmosphere. J. Phys. Oceanogr., 49, 385-407, https://doi.org/10.1175/JPO-D-18-0092.1.
Lvov, Y. V., K. L. Polzin, and E. G. Tabak, 2004: Energy spectra of the ocean's internal wave field: Theory and observations. Phys. Rev. Lett., 92, 128501, http://doi.org/10.1103/physrevlett.92.128501.

McComas, C., and P. Muller, 1981a: The dynamic balance of internal waves. J. Phys. Oceanogr., 11, 970-986, https://doi.org/ 10.1175/1520-0485(1981)011<0970:TDBOIW>2.0.CO;2.

—_, and —_, 1981b: Time scales of resonant interactions among oceanic internal waves. J. Phys. Oceanogr., 11, 139-147, https://doi.org/10.1175/1520-0485(1981)011<0139: TSORIA $>2.0 . \mathrm{CO} ; 2$.

Müller, P., and D. Olbers, 1975: On the dynamics of internal waves in the deep ocean. J. Geophys. Res., 80, 3848-3860, https:// doi.org/10.1029/JC080i027p03848.

— E. E. D'Asaro, and G. Holloway, 1992: Internal gravity waves and mixing. Eos, Trans. Amer. Geophys. Union, 73, 25 and 31-32, http://doi.org/10.1029/91eo00018.

Munk, W. H., 1981: Internal waves and small-scale processes. Evolution of Physical Oceanography, B. A. Warren and C. Wunsch, Eds., MIT Press, 264-291.

Orlanski, I., and K. Bryan, 1969: The formation of thermocline step structure by large-amplitude internal gravity waves. J. Geophys. Res., 74, 6975-6983, https://doi.org/10.1029/ JC074i028p06975.

Pinkel, R., 2014: Vortical and internal wave shear and strain. J. Phys. Oceanogr., 44, 2070-2092, https://doi.org/10.1175/ JPO-D-13-090.1.

- 2020: The Poisson link between internal wave and dissipation scales in the thermocline. Part I: Probability density functions and the Poisson modeling of vertical strain. J. Phys. Oceanogr., 50, 3403-3424, https://doi.org/10.1175/JPO-D-19-0286.1.

_- , and S. Anderson, 1992: Toward a statistical description of finescale strain in the thermocline. J. Phys. Oceanogr., 22, 773-795, https://doi.org/10.1175/1520-0485(1992)022<0773: TASDOF $>2.0 . \mathrm{CO} ; 2$.

Polzin, K. L., J. M. Toole, and R. W. Schmitt, 1995: Finescale parameterizations of turbulent dissipation. J. Phys. Oceanogr., 25, 306-328, https://doi.org/10.1175/1520-0485(1995)025<0306: FPOTD $>2.0 . C O ; 2$.

- A. C. N. Garabato, T. N. Huussen, B. M. Sloyan, and S. Waterman, 2014: Finescale parameterizations of turbulent dissipation. J. Geophys. Res. Oceans, 119, 1383-1419, https:// doi.org/10.1002/2013JC008979.

Stommel, H., and K. N. Fedorov, 1967: Small scale structure in temperature and salinity near Timor and Mindanao. Tellus, 19, 306-325, https://doi.org/10.3402/tellusa.v19i2.9792.

Sun, H., and E. Kunze, 1999: Internal wave-wave interactions: Part I. The role of internal wave vertical divergence. J. Phys. Oceanogr., 29, 2886-2904, https://doi.org/10.1175/1520-0485(1999)029<2886: IWWIPI $>2.0 . \mathrm{CO} ; 2$.

Thorpe, S. A., 1977: Turbulence and mixing in a Scottish loch. Philos. Trans. Roy. Soc. London, A286, 125-181, https:// doi.org/10.1098/rsta.1977.0112.

Whalen, C. B., L. D. Talley, and J. A. MacKinnon, 2012: Spatial and temporal variability of global ocean mixing inferred from Argo profiles. Geophys. Res. Lett., 39, L18612, https://doi.org/ 10.1029/2012GL053196.

Winters, K. B., and E. A. D'Asaro, 1994: Three-dimensional wave instability near a critical level. J. Fluid Mech., 272, 255-284, https://doi.org/10.1017/S0022112094004465. 